\title{
Reduction Over Time: Easing the Burden of Peer-to-Peer Barter Relationships to Facilitate Mutual Help
}

\author{
Kenji Saito ${ }^{1}$, Eiichi Morino ${ }^{2}$, and Jun Murai ${ }^{3}$ \\ 1 Graduate School of Media and Governance, Keio University \\ ks91@sf c.wide.ad.jp \\ 2 Gesell Research Society Japan \\ ${ }^{3}$ Faculty of Environmental Information, Keio University
}

\begin{abstract}
A peer-to-peer complementary currency can be a powerful tool for promoting exchanges and building relationships for coordinated activities. $i$-WAT[11] is a proposed such currency usable on the Internet. It is based on the WAT System[15], a polycentric complementary currency using WAT tickets as its media of exchange: participants spontaneously issue and circulate the tickets as needed, whose values are backed up by chains of trust. $i$-WAT implements the tickets electronically by exchanging messages signed in OpenPGP[3].

This paper proposes an extension to the design of $i$-WAT to facilitate mutual help among people in need. In particular, we propose additional "reduction" tickets whose values are reduced over time. By deferring redemption of such tickets, the participants can contribute to reduce the debts of the issuers, as well as to accelerate spending. Applications of this feature include a relief to disaster-affected people.

A reference implementation of $i$-WAT has been developed in the form of a plug-in for an XMPP[6][7] instant messaging client. We have been putting the currency system into practical use, to which the proposed feature will be added shortly.
\end{abstract}

\section{Introduction}

\subsection{Peer-to-Peer Complementary Currency}

One of the issues of activity coordination is incentive-compatibility[8], roughly restated as the goal of the coordinated activity being accomplished by collection of selfish behaviors. Because it necessitate fair exchanges of resources among participants, the medium of exchange must take an important role.

Money is a well-known medium of exchange, but its scarcity has caused a lot of problems. Complementary currencies, or alternative forms of monetary media, have been proposed and tested to achieve an autonomous, sustainable local economy even in short of money. There have been successful cases, such as experiments in Wörgl in 1932 (stamp money[13]), in Comox Valley in 1983 (Local Exchange Trading System[14]) and in Ithaca since 1991 (Ithaca HOURs[10]). 
Many of those outcomes are short-lived, however, because most of the existing currencies are dependent on the qualities of their administrations. It would thus benefit the autonomy and sustainability of economy if we could design an administration-free (or peer-to-peer) complementary currency.

We proposed $i$-WAT[11] in year 2003 as such a currency usable on the Internet, based on the WAT System[15]. The WAT System is a system of polycentric complementary currencies using WAT tickets as its media of exchange. A WAT ticket is like a bill of exchange, but without a specified redemption date or place. $i$-WAT implements the tickets electronically by exchanging messages signed in OpenPGP[3]. It has been put into practical use since June 2004.

\subsection{Reduction Over Time to Accelerate Spending}

It is known among the practitioners of complementary currencies that reducing the value of the exchange medium over time accelerates spending. The stamp money experiment in Wörgl in 1932 is a well-known example. It was based on the idea of stamp scrip introduced by Sylvio Gesell in [9], who believed exchange media must also deteriorate as the exchanged goods do (for this reason, Reduction Over Time is dubbed ROT hereafter).

A user of stamp money needs to paste a stamp every week on the back of the note, or the note becomes invalid. Users are motivated to spend the scrip before another stamp is required. The stamp is a tax for storage of exchange medium. It is a suitable way to implement ROT in a centralized currency system.

\subsection{Contributions of This Paper}

If ROT is to be implemented for decentralized currencies, a different approach needs to be taken. We have applied the notion of calendar money[5] by Arthur Dahlberg, which has a schedule of reduction printed on the note.

We have realized that this has potential effects of not only promoting exchanges, but also providing participants with means to support peers by sharing debts among one another.

This paper describes WAT/ $i$-WAT concisely, and proposes an extension to the design of $i$-WAT to realize the above concept. It shows that the extended design is incentive-compatible by a casual analysis.

\section{WAT/ $i$-WAT Currency System}

\subsection{The WAT System}

Overview The WAT System[15] is a complementary currency designed by Eiichi Morino, a coauthor of this paper. It has been used broadly as a means for mediating bartering, since its introduction in August 2000.

A WAT ticket, a physical sheet of paper resembling a bill of exchange, is used as the medium of exchange in the system. A lifecycle of a WAT ticket involves three stages of trading as illustrated in Fig. 1: 


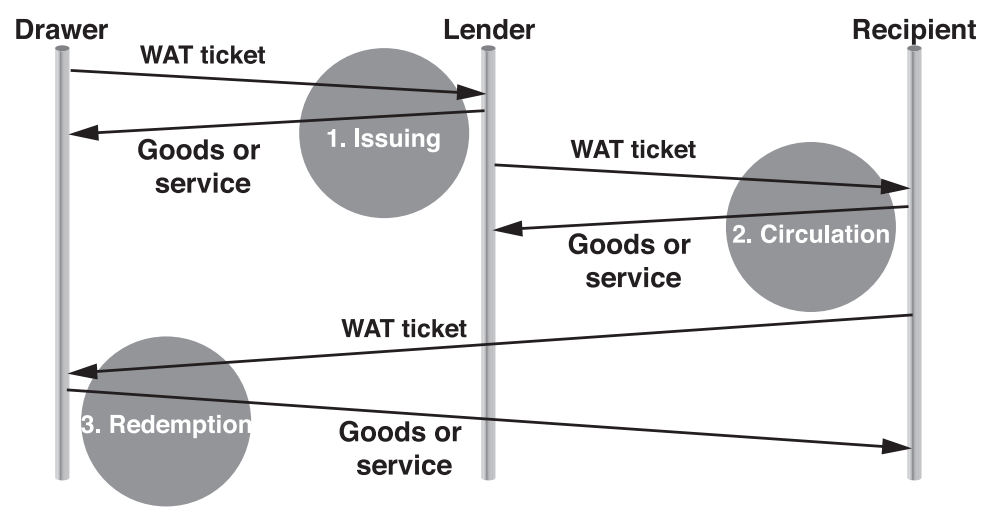

Fig. 1. Three stages of trading with a WAT ticket

1. Issuing - the birth of a WAT ticket

A drawer issues a WAT ticket by writing on an empty form the name of the provider (lender) of the goods or service, the amount of debt ${ }^{1}$, the present date, and the drawer's signature. The drawer gives the ticket to the lender, and in return obtains some goods or service.

2. Circulation - ordinary exchange

The person to whom the WAT ticket was given can become a user, and use it for another trading. To do so, the user writes the name of the recipient, as well as their own, on the reverse side of the ticket. The recipient will become a new user, repeating which the WAT ticket circulates among people.

3. Redemption - the return of the WAT ticket

The WAT ticket is invalidated when it returns, as a result of a trade, to the drawer.

\section{Distinctive Features of the WAT System}

Autonomy Anyone can spontaneously become a member of the WAT System with a sheet of paper if they follow the above protocol.

Compatibility A WAT ticket is compatible with any other WAT tickets in the world, so that the currency system is operable globally, as long as the drawer can be credited.

Extensibility The protocol illustrated in Fig. 1 defines the WAT Core, the essence of the WAT System. An extended part can be defined for a new currency based on the WAT System, stating, for example, the region, group and duration in which the tickets are usable, as well as the unit in which the debt is quantified.

\footnotetext{
${ }^{1}$ Typically in the unit kWh, which represents cost of producing electricity from natural
} energy sources. 
Table 1. $i$-WAT messages

\begin{tabular}{|l|l|l|l|}
\hline \multicolumn{1}{|c|}{ message } & sender & \multicolumn{1}{c|}{ receiver } & \multicolumn{1}{c|}{ function } \\
\hline$<$ draw $/>$ & drawer & recipient (lender) & draws an $i$-WAT ticket. \\
\hline$<$ use $/>$ & user & recipient & uses an $i$-WAT ticket. \\
\hline$<$ accept $/>$ & recipient & drawer and user & $\begin{array}{l}\text { confirms readiness to accept the } i \text {-WAT } \\
\text { ticket once it is validated. }\end{array}$ \\
\hline$<$ reject $/>$ & recipient & drawer or user* & rejects an $i$-WAT ticket. \\
\hline$<$ approve $/>$ & drawer & user and recipient & $\begin{array}{l}\text { validates an } i \text {-WAT ticket, and ap- } \\
\text { proves the transaction. }\end{array}$ \\
\hline$<$ disapprove $/>$ & drawer & user and recipient & denies an $i$-WAT transaction. \\
\hline
\end{tabular}

Security In case the drawer fails to meet their promise on the ticket, the lender assumes the responsibility for the debt. If the lender fails, the next user takes over. The responsibility follows the chain of endorsements. The longer the chain is, the more firmly backed up the ticket is. Therefore the length of the chain of endorsements represents the extent of trust the ticket has gained.

\section{2 i-WAT: the Internet WAT System}

Overview $i$-WAT is a translation of the WAT Core onto the Internet. We have made a reference implementation available to the public, which has been used mainly by the members of the WAT System.

In $i$-WAT, messages signed in OpenPGP ( $i$-WAT messages) are used to implement transfers of an electronically represented WAT ticket ( $i$-WAT ticket).

An $i$-WAT ticket contains the identification number, amount of debt and public key user IDs of the drawer, users and recipients. Endorsements are realized by nesting PGP signatures.

Table 1 shows the types of $i$-WAT messages. All $i$-WAT messages are signed by the senders, and are formatted in the canonical form[1] of XML[2] with nested signatures. The messages cause state transfers of a ticket as illustrated in Fig. 2.

Changes from the WAT System Upon translating the WAT Core onto the digital communication domain, we have made the following changes from the state machine of a WAT ticket:

1. Trades need to be asynchronously performed. Intermediate states, such as waiting for acceptance or approval, are introduced.

2. Double-spending needs to be prohibited. The drawer is made responsible for guaranteeing that the circulating ticket is not a fraud. This means that every trade has to be approved by the drawer of the involved ticket.

The semantics of this design and the trust model of $i$-WAT are discussed in detail in [12]. 


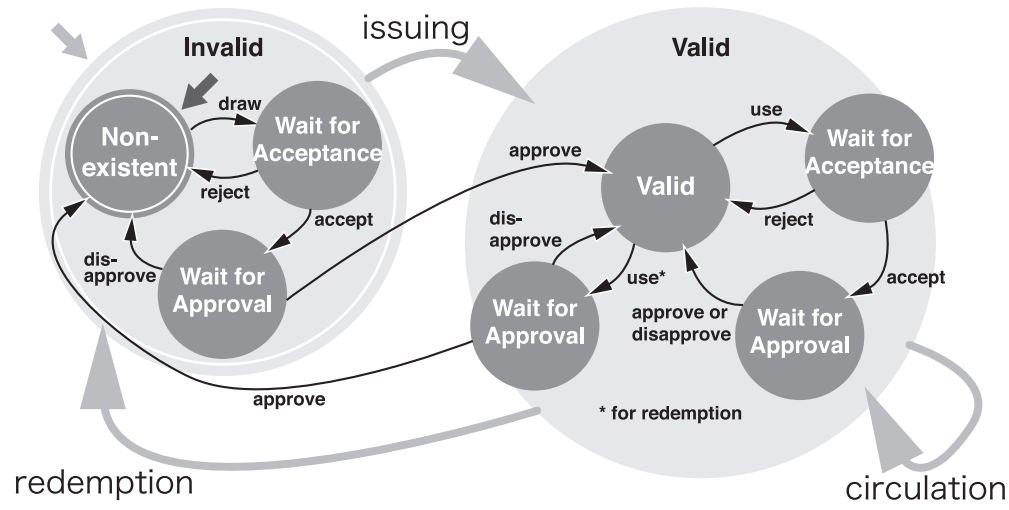

* Gray arrows represent WAT state-transfer.

* Black arrows represent $i$-WAT state-transfer.

Fig. 2. State machine of a WAT $/ i$-WAT ticket

\section{Protocol}

Issuing - the birth of an i-WAT ticket (Fig. 3)

1. The drawer sends a $<$ draw/ $>$ message which contains the public key user IDs of the drawer and lender, identification number and amount of debt. This message becomes the original $i$-WAT ticket after the protocol is completed.

2. The lender sends back the content of the message as an $<$ accept $/>$ message.

3. The drawer sends an <approve/> message to the lender.

\section{Circulation - ordinary exchange (Fig. 4)}

1. The user adds to the $i$-WAT ticket the public key user ID of the recipient, and sends it to the recipient as a $<$ use/ $>$ message. This message becomes a valid $i$-WAT ticket after the protocol is completed.

2. The recipient forwards the content of the message to the drawer and user as an $<$ accept $/>$ message.

3. The drawer verifies the ticket, and sends an <approve/> message to the user and recipient.

Redemption - the return of the $i$-WAT ticket (Fig. 5)

1. The user sends a $<$ use/ $>$ message to the recipient, who equals the drawer.

2. The drawer verifies the ticket, and invalidates it as the debt is now redeemed. The drawer sends an <approve/> message to the user. 


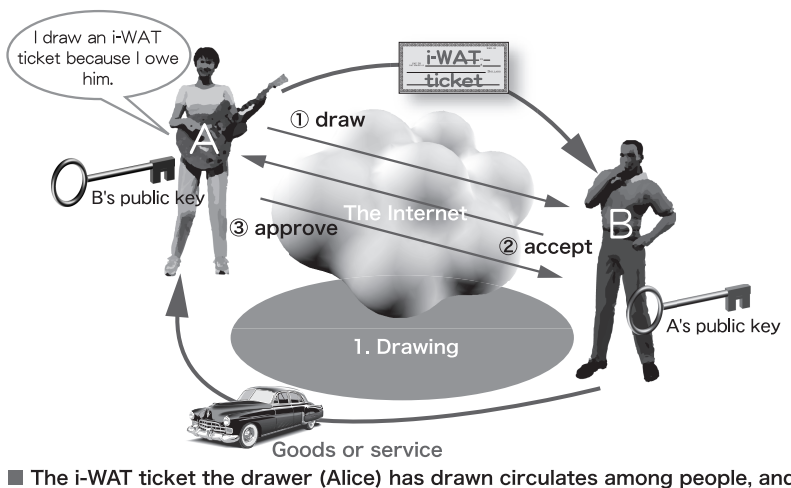

The i-WAT ticket the drawer (Alice) has gets redeemed when it returns to her.

Fig. 3. $i$-WAT transaction 1: issuing

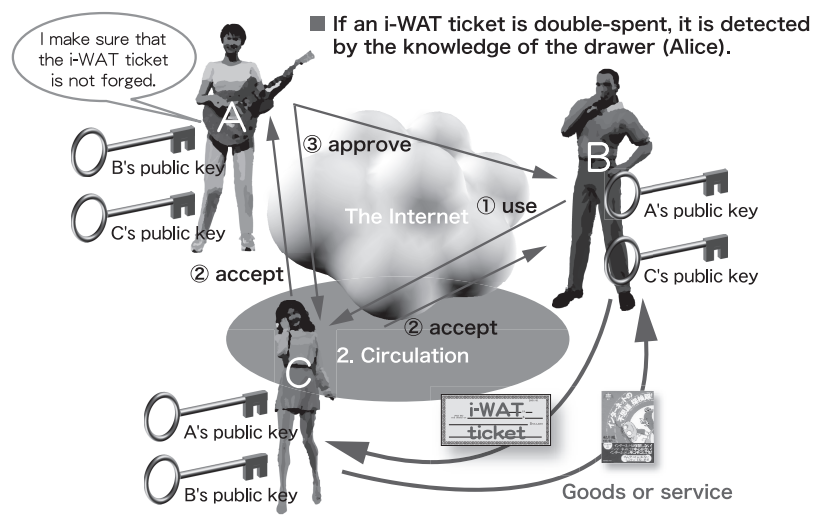

Fig. 4. $i$-WAT transaction 2: circulation

\section{ROT: Reduction Over Time}

\subsection{Concept}

We make a generalization to the value of a WAT $/ i$-WAT ticket such that it is expressed as a tuple $\left\langle V_{0}, V_{m}, f\right\rangle$ presented by the drawer, where $V_{0}$ is the face value (initial value) of the ticket, $V_{m}$ is the minimum value, and $f(t)$ is the differentiation (derivative) of a function of time $F(t)$ such that $f(t) \leq 0$ for all $t$ (an extension to allow $f(t)>0$ is conceivable, but it will be discussed elsewhere). The effective value $V_{t}$ of a ticket at time $t$ is given by the following equation:

$$
V_{t}=\max \left(\int_{0}^{t} f(t) d t+V_{0}, V_{m}\right)
$$




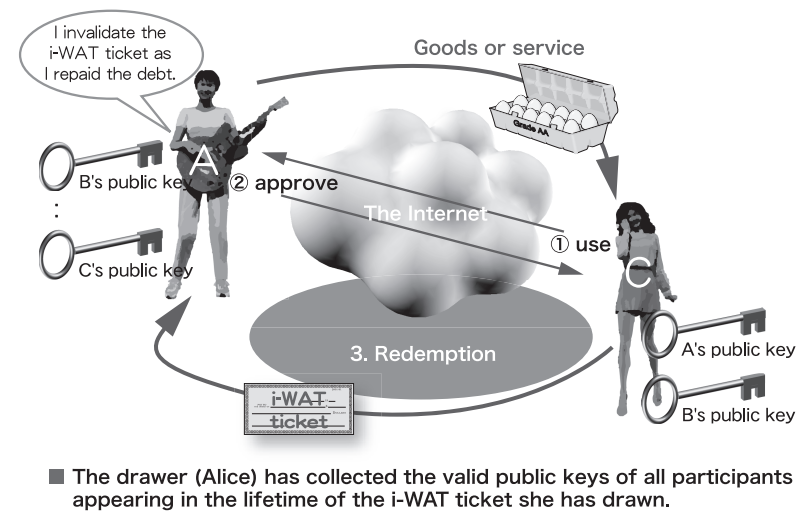

Fig. 5. $i$-WAT transaction 3: redemption

This is a generalization to introduce an additional type of tickets called reduction tickets, whose values are reduced over time, limited by a minimum value. For regular WAT $/ i$-WAT tickets, $f(t)$ is a constant value zero, and $V_{0}=V_{m}$.

Reduction of the value of a ticket means that the drawer's debt is reduced. The cost of reduction is first admitted by the lender who credits the drawer, and then shared among the endorsers as illustrated in Fig. 6. The amount of the total reduction is manifested to the drawer upon redemption. By deferring redemption, participants can help easing the burden of the drawer.

\subsection{Incentive-Compatibility of the Design}

We show that the design of reduction tickets is incentive-compatible by a casual analysis.

Lender's Reasoning The lender may not want to accept a reduction ticket because they know that its value will decrease over time if they save it. They can be motivated to accept the ticket, however, if they know that the drawer is in need, such as in a case of aftermath of a natural disaster. They know that they do not have to take all the burden by themselves; they infer that, by the same reasoning as theirs, it is likely that those people around them are also willing to accept the ticket. Therefore the ticket is accepted, and spent as soon as possible to satisfy the need of the lender themselves.

Endorser's Reasoning All participants, if they are aware of the situation of the drawer, will be motivated to:

1. defer redemption of the ticket to support the drawer, and

2. spend it as soon as possible to avoid much reduction of its value and to satisfy their own needs. 


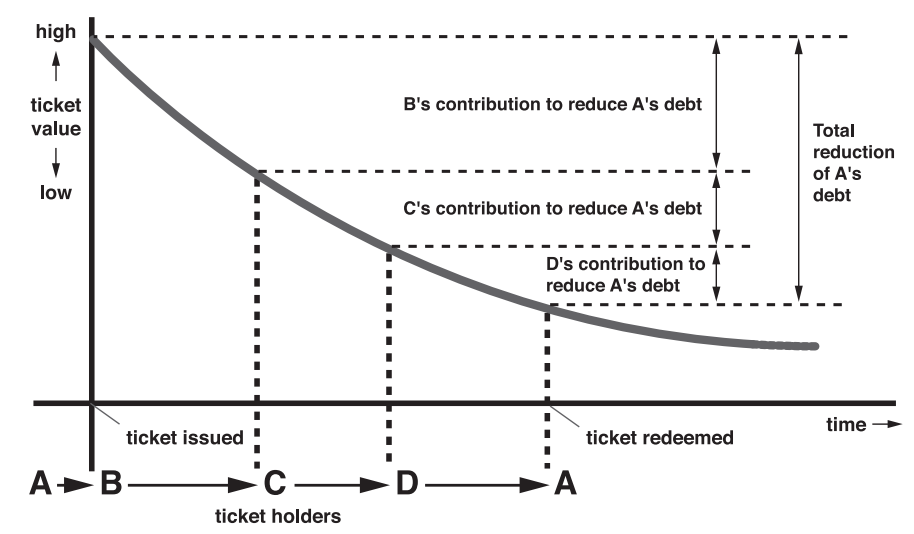

Fig. 6. Giving relief by deferring redemption of a reduction ticket

We predict that a reduction ticket will typically circulate at high speed until its effective value reaches the minimum value $V_{m}$.

Drawer's Reasoning There is a risk that circulation may be stalled for an $i$ WAT ticket by negligence of the drawer in their role for approving transactions. However, we can show that it is to the drawer's own benefit to maintain the liveness of their tickets:

1. If one is late to respond, or tends to fail to answer requests for redemption, they will lose trust from others. Then it will become more difficult for them to have their tickets accepted for future trades.

2. If one is quick to respond, and accepts requests for redemption with certainty, they will gain more trust from others. Since their tickets become easier to use, they may attract more load. But it will become easier for them to draw tickets at will in the future, and initiate trades spontaneously to obtain goods or services.

\subsection{Protocol}

Reduction tickets can be incorporated to the system with minimal changes to the existing $i$-WAT protocol.

Since it is unrealistic to assume that the clocks of all participating computers are synchronized with precision, the time is ultimately measured by the computer of the drawer.

The timestamp of the drawer's or user's signature (depending on whether the trade is issuing or circulation) defines the effective value of a reduction ticket, to which the recipient either agrees or disagrees. The drawer (or their software agent) is responsible to check that the timestamp belongs to the past for them when they approve a transaction. 


\subsection{Applications}

Applications of reduction tickets include relief to disaster-affected people.

We are sure that everyone is hurt by the tsunami which swept the coastlines of Southeast Asian countries on December 26, 2004, killed an unprecedented number of people and took means of life away from literally millions.

We have made a proposal to $c c$ Tsunami[4], which is an open forum on the Internet for discussing and implementing programs to support the tsunami-affected places with complementary currencies. In the proposal, $i$-WAT tickets are issued by an NGO which promises to exchange the tickets with WAT tickets issued by the disaster-affected people, so that people in need do not need to use computers.

\section{Ongoing and Future Works}

\subsection{Implementation}

We have been developing a reference implementation of $i$-WAT as a plug-in for wija, an XMPP (Extensible Messaging and Presence Protocol)[6][7] messaging client. Although we cannot quantify the number of users because of the nature of the WAT System, many who have experienced the physical WAT System are using $i$-WAT despite of technical difficulties they may be suffering. We are in a continous process of improving the ease of use of the system.

Implementation of the proposed ROT feature is in progress. It will be included in the bundled plug-in for the new version of wija to be released in March 2005.

wija is available at http://www.media-art-online.org/wija/.

\subsection{Formal Analysis}

We are working on a more formal analysis to show that the ROT feature is incentive-compatible. A partial result shows that an equilibrium is achieved when the scheduled minimum value of a reduction ticket is zero. Although this is compatible with everyone's benefit, it may cause a moral hazard such that the drawer issues as many tickets as they want. We are pursuing an operational design which mitigates this hazard.

\subsection{Future Work}

Once the new implementation is ready, we will experiment on actual usage of the ROT feature to verify the prediction that the reduction tickets typically circulate at high speed until their effective values hit the minimum. 


\section{Conclusions}

This paper proposed an extension to the design of $i$-WAT to implement reduction over time, which has potential effects of both promoting exchanges and providing participants with means to support peers, by sharing debts among one another as a form of currency. The extended design is shown to be incentive-compatible by a casual analysis. The implementation will be available to the public shortly.

\section{References}

1. John Boyer. Canonical XML Version 1.0, March 2001. W3C Recommendation. Available electronically at http://www.w3.org/TR/xml-c14n.

2. Tim Bray, Jean Paoli, C.M.Sperberg-McQueen, and Eve Maler. Extensible Markup Language (XML) 1.0 (Second Edition), October 2000. W3C Recommendation. Available electronically at http://www.w3.org/TR/REC-xml.

3. Jon Callas, Lutz Donnerhacke, Hal Finney, and Rodney Thayer. OpenPGP Message Format, November 1998. RFC 2440.

4. ccTsunami.org. Complementary currency tsunami relief center. Hypertext document. Available electronically at http://www.cctsunami.org/.

5. Arthur Dahlberg. When Capital Goes On Strike. Harper \& Brothers Publishers, 1938.

6. Peter Saint-Andre (Ed). Extensible Messaging and Presence Protocol (XMPP): Core, October 2004. RFC 3920.

7. Peter Saint-Andre (Ed). Extensible Messaging and Presence Protocol (XMPP): Instant Messaging and Presence, November 2004. RFC 3921.

8. Joan Feigenbaum and Scott Shenker. Distributed algorithmic mechanism design: Recent results and future directions. In Proceedings of the 6th International Workshop on Discrete Algorithms and Methods for Mobile Computing and Communication (DIALM '02), September 2002.

9. Sylvio Gesell. The Natural Economic Order. The Free Economy Publishing Co., 1934. Translated from the sixth German edition (originally published in 1913). Also available as a hypertext document in English, translated by Phillip Pye, at http://www.systemfehler.de/en/neo/.

10. Paul Glover. Ithaca HOURs Online. Hypertext document. Available electronically at http://www.ithacahours.com/.

11. Kenji Saito. Peer-to-peer money: Free currency over the Internet. In Proceedings of the Second International Conference on Human.Society@Internet (HSI 2003), Lecture Notes in Computer Science 2713. Springer-Verlag, June 2003.

12. Kenji Saito. WOT for WAT: Spinning the web of trust for peer-to-peer barter relationships. In IEICE TRANSACTIONS on Communication. The Institute of Electronics, Information and Communication Engineers, April 2005 (to appear).

13. Fritz Schwarz. Das experiment von Wörgl, 1951. Hypertext document. Available electronically at http://userpage.fu-berlin.de/ roehrigw/woergl/, (Shortened English translation by Hans Eisenkolb is available at http://www.sunshinecable.com/ eisehan/woergl.htm).

14. Sidonie Seron. Local Exchange Trading Systems 1 - CREATION AND GROWTH OF LETS. Hypertext document. Available electronically at http://www.gmlets.u-net.com/resources/sidonie/ home.html.

15. watsystems.net. WATSystems home page. Hypertext document. Available electronically at http://www.watsystems.net/. 